OPEN ACCESS

Edited by:

Peng Xu,

Xiamen University, China

Reviewed by:

Yamei Xiao,

Hunan Normal University, China

Deshou Wang,

Southwest University, China

${ }^{*}$ Correspondence:

Tiansheng Chen

tiansheng.chen@mail.hzau.edu.cn

Ruibin Yang

rbyang@mail.hzau.edu.cn

tThese authors have contributed equally to this work.

¥ORCID:

Tiansheng Chen orcid.org/0000-0003-4763-2307

Ruibin Yang orcid.org/0000-0003-0888-6347

Specialty section:

This article was submitted to

Livestock Genomics,

a section of the journal

Frontiers in Genetics

Received: 28 February 2019

Accepted: 03 July 2019

Published: 02 August 2019

Citation:

Bian F, Yang X, Ou Z, Luo J, Tan B, Yuan M, Chen T and Yang $R$ (2019) Morphological Characteristics and

Comparative Transcriptome Analysis of Three Different Phenotypes of

Pristella maxillaris.

Front. Genet. 10:698.

doi: 10.3389/fgene.2019.00698

\section{Morphological Characteristics and Comparative Transcriptome Analysis of Three Different Phenotypes of Pristella maxillaris}

\author{
Fangfang Bian ${ }^{1 \dagger}$, Xuefen Yang ${ }^{1 \dagger}$, Zhijie Ou ${ }^{1,2}$, Junzhi Luo ${ }^{1}$, Bozhen Tan ${ }^{1}$, Mingrui Yuan ${ }^{1}$, \\ Tiansheng Chen ${ }^{1,3 \neq *}$ and Ruibin Yang ${ }^{1 \neq *}$
}

${ }^{1}$ Key Laboratory of Freshwater Animal Breeding, Ministry of Agriculture, College of Fisheries, Huazhong Agricultural University, Wuhan, China, ${ }^{2}$ Department of Fisheries, Guangdong Maoming Agriculture \& Forestry Technical College, Maoming, China, ${ }^{3}$ Collaborative Innovation Center for Efficient and Health Production of Fisheries in Hunan Province, Changde, China

Pristella maxillaris is known as the X-ray fish based on its translucent body. However, the morphological characteristics and the molecular regulatory mechanisms of these translucent bodies are still unknown. In this study, the following three phenotypes, a black-and-gray body color or wild-type (WT), a silvery-white body color defined as mutant I (MU1), and a fully transparent body with a visible visceral mass named as mutant II (MU2), were investigated to analyze their chromatophores and molecular mechanisms. The variety and distribution of pigment cells in the three phenotypes of $P$. maxillaris significantly differed by histological assessment. Three types of chromatophores (melanophores, iridophores, and xanthophores) were observed in the WT, whereas MU1 fish were deficient in melanophores, and MU2 fish lacked melanophores and iridophores. Transcriptome sequencing of the skin and peritoneal tissues of $P$. maxillaris identified a total of 166,089 unigenes. After comparing intergroup gene expression levels, more than 3,000 unigenes with significantly differential expression levels were identified among three strains. Functional annotation and Gene Ontology (GO) and Kyoto Encyclopedia of Genes and Genomes (KEGG) pathway analyses of the differentially expressed genes (DEGs) identified a number of candidates melanophores and iridophores genes that influence body color. Some DEGs that were identified using transcriptome analysis were confirmed by quantitative real-time PCR. This study serves as a global survey of the morphological characteristics and molecular mechanism of different body colors observed in P. maxillaris and thus provides a valuable theoretical foundation for the molecular regulation of the transparent phenotype.

Keywords: Pristella maxillaris, RNA-seq, melanophores, iridophores, molecular mechanism

\section{INTRODUCTION}

As one of the most diverse phenotypic traits under strong selection pressure in many organisms, coloration plays numerous adaptive functions such as predator deterrence, species recognition, and even protecting the organism from solar ultraviolet radiation damage (Lowe and Goodman-Lowe, 1996; Parichy, 2006; Roberts et al., 2009; Zhang et al., 2015). Skin coloration can be influenced by 
many factors, such as genetics, diet, and general health (Wang et al., 2014; Zhang et al., 2015). Nevertheless, genetics remains the major determining factor (Braasch et al., 2007). Most animals have different body colors that are mainly determined by diverse pigments synthesized by chromatophores or pigment cells. Chromatophores are cells that are specialized in the storage and/or synthesis of light-absorbing pigments or light-reflecting structures (Bagnara et al., 2007; Leclercq et al., 2010; Henning et al., 2013). Teleost fishes have more than six types of pigment cells (Michiels et al., 2008; Wucherer and Michiels, 2012; Goda et al., 2013; Wucherer and Michiels, 2014; Schartl et al., 2016). Zhang et al. (2015) found that the differences in two color patterns of the crimson snapper (Lutjanus erythropterus) primarily depended on the density and distribution of pigment cells; in black skin, melanophores are the major pigment cells, and in red skin, iridophores and xanthophores are the major pigment cells. In adult red crucian carp (Carassius auratus, red var.), body color undergoes a gray-to-red change, which is due to alterations in the number of skin melanophores (Zhang et al., 2017). In addition, several studies have reported that many fish species can change body transparency based on the differentiation and development of chromatophores (Nilsson Skold et al., 2010; Krauss et al., 2013; Franco-Belussi et al., 2016). Some model fish mutants, such as medaka and zebrafish, exhibit a transparent phenotype by regulating the expression levels of some genes (Parichy, 2006; White et al., 2008; Krauss et al., 2013; Kimura et al., 2017). The differentiation and development of pigment cells are strictly regulated by genes (Roberts et al., 2009; Dooley et al., 2013), whereas the genetics behind natural color morph variants in fish remains largely unknown.

RNA-Seq analysis is the most convenient method to investigate gene expression patterns in organisms. To date, several studies have revealed gene expression profiles that are responsible for different color patterns in freshwater fish. The striped pattern of the zebrafish (Danio rerio) is generated by self-organizing mechanisms that require interactions among three different types of pigment cells (Irion et al., 2016). In addition, transcriptome analyses of different colored varieties of the common carp (Cyprinus carpio var. color) (Wang et al., 2014), crimson snapper (Zhang et al., 2015), Midas cichlid (Amphilophus citrinellus) (Higdon et al., 2013), and red crucian carp (Zhu et al., 2018) were performed to understand the genetic basis of coloration. Signaling pathways, such as the Wnt $/ \beta$ catenin (wingless-type MMTV integration site family), tyrosinase synthesis, MAPK (mitogen-activated protein kinase), and cAMP (cyclic adenosine monophosphate) pathways, have been shown as conserved pathways that are related to melanophore development in vertebrates (Jiang et al., 2014; Wang et al., 2014). Several studies have investigated the regulatory mechanism of melanophore development, and many pigment-related genes have been identified in mice and fish (Newton et al., 2005; Hoekstra et al., 2006; Fang et al., 2018). However, only a few studies have investigated the role of iridophores (Ng et al., 2009; Higdon et al., 2013) and xanthophores (Sefc et al., 2014) in body coloration, and their detailed molecular mechanism has been less investigated.

Pristella maxillaris, also known as the X-ray fish, is a warm water fish belonging to the family Characidae and the order
Characiformes. It is a widely distributed and adaptable fish found in the Amazon and Orinoco basins, as well as in coastal rivers in the Guianas. Due to its translucent body color, $P$. maxillaris is a valuable, ornamental fish that has a huge market. Morphological diversification of $P$. maxillaris has produced many kinds of transparent body mutations leading to coloration ranging from a black-gray body color to entirely transparent. There are three typical phenotypes: wild-type (WT), which has a black-and-gray body color with black spots on the trailing edge and fin of the operculum; mutant I (MU1), which has a silvery-white body color; and mutant II (MU2), which is fully transparent and has clearly observed visceral tissues. To date, most studies on $P$. maxillaris have mainly focused on their growth and development (Yu et al., 2018). However, investigations on body transparency mutations and the molecular mechanism in $P$. maxillaris have not yet been conducted. To better understand how cells and genetic factors alter body transparency, we utilized stereomicroscopy to observe the differences in chromatophores in WT fish and two different mutants, namely, MU1 and MU2. RNA-Seq was conducted on samples from the three phenotypes to compare their gene expression profiles. In particular, the signaling pathways and candidate genes whose mutations are responsible for differences in body transparency were also examined and quantified. The purpose of this study was to provide a global survey of the morphological characteristics and molecular mechanism of the different body colors in $P$. maxillaris, as well as generate theoretical foundation for the molecular regulation of the transparent phenotype.

\section{MATERIALS AND METHODS}

\section{Ethics Statement}

No specific permissions were required for the use of $P$. maxillaris collected for this study in China. All the experimental procedures involving fish were approved by the Institutional Animal Care and Use Committee of Huazhong Agricultural University.

\section{Samples for Microscopy and Transcriptome Analysis}

Samples from fish exhibiting three $P$. maxillaris phenotypes (WT, MU1, and MU2) were collected from the Flower and Bird Market in Wuhan, Hubei, China. Prior to the experiments, the fish were kept in laboratory aquariums under 14:10 h light/dark conditions at temperatures of $24 \pm 2{ }^{\circ} \mathrm{C}$ for 2 weeks to acclimate them to the experimental conditions. The fish were anesthetized in well-aerated water containing $100 \mathrm{mg} / \mathrm{L}$ tricaine methanesulfonate (MS-222) before being immediately euthanized. Six adult individuals exhibiting each $P$. maxillaris phenotype (average length, $3.5 \pm 0.3 \mathrm{~cm}$ ) were selected. The fresh pieces of the operculum lining, peritoneum, and skin were surgically excised and temporarily mounted for subsequent light microscopic (OLYMPUS SZX16) observation. In addition, fish exhibiting the three phenotypes were anesthetized and fixed for $24 \mathrm{~h}$ in formalin, and areas of approximately $1 \mathrm{~cm}^{2}$ in size were cut from the skin and peritoneal tissues for paraffin sectioning. 
The types and distributions of pigment cells in fish exhibiting the three different phenotypes were observed under a microscope (Imager A2). Pigment cell types are easily identified due to their colors and shapes by microscopic and histological methods based on the literatures. Melanophores show black/gray color and stellated shape; xanthophores exhibit yellow, orange, and red colors; iridophores contain white, blue, and purple-red color (Kelsh, 2004; Darias et al., 2013; Zhang et al., 2015; Zhang et al., 2017).

Additional skin and peritoneal tissues from different phenotypes were collected to extract total RNA, and we pooled the skin and peritoneal tissues from multiple individuals of each phenotype of $P$. maxillaris. All fresh tissue samples were frozen immediately in liquid nitrogen and then stored at $-80^{\circ} \mathrm{C}$ before RNA isolation.

\section{RNA Extraction}

Total RNA was obtained from the mixed samples of skin and peritoneum from fish exhibiting the three different phenotypes of P. maxillaris using RNAiso Plus Reagent (TaKaRa, China) according to the manufacturer's protocol. Total RNA was extracted with a Qubit ${ }^{\circledR}$ RNA Assay Kit in a Qubit ${ }^{\circledR}$ 2.0 Fluorometer (Life Technologies, CA, USA). The RNA Nano 6000 Assay Kit from the Agilent Bioanalyzer 2100 system (Agilent Technologies, CA, USA) and gel electrophoresis were used to assess the quantity and quality of the total RNA.

mRNA was purified from the total RNA using polyT oligoattached magnetic beads (NEB, USA). Fragmentation was carried out using divalent cations under elevated temperature in NEB-Next First-Strand Synthesis Reaction Buffer $(5 \times)$. Firststrand cDNA was synthesized using random hexameric primers and M-MuLV reverse transcriptase (RNase $\mathrm{H}$ ). Second-strand cDNA synthesis was subsequently performed using DNA polymerase I and RNase $\mathrm{H}$. The remaining overhangs were converted into blunt ends via exonuclease/polymerase activities. After adenylation of the 3' ends of the DNA fragments, NEB Next Adaptor with hairpin loop structure was ligated to prepare for hybridization. cDNA fragments 250 to 300 bp long were selected as templates. An Agilent 2100 Bioanalyzer (Agilent Technologies, Santa Clara, CA, USA) and an AMPure XP real-time PCR system (Beckman Coulter, Beverly, USA) were used to quantify and qualify the sample library.

\section{Sequencing, Assembly, and Annotation}

Transcriptome sequencing was conducted on an Illumina HiSeq 2000 RNA-Seq platform (Illumina, San Diego, CA, USA). Clean reads were acquired after removing reads with adapters, reads with more than 5\% unknown nucleotides, and reads with a percentage of low-quality bases (base quality d' 10) more than $20 \%$. Trinity was used to conduct the de novo assembly of the transcriptome (Grabherr et al., 2011). Contigs, longer fragments without $\mathrm{N}$, were obtained by combining overlapping reads. Then, different contigs were connected to obtain sequences that could not be extended on either end, which were defined as unigenes. The assembled sequences were compared against the NCBI nonredundant $(\mathrm{Nr})$ protein database, Swiss-Prot, Kyoto Encyclopedia of Gene and Genomes (KEGG), and the Clusters of Orthologous Groups (COG) database using BLASTX with an E-value of $1 \times$ $10^{-5}$. The directions of the contig sequences were based on the best alignment results. A combination of the BLAST, Blast2GO, KEGG, and GO databases was used for functional annotation. BLASTX alignment (E-value $<1 \times 10^{-5}$ ) with the NT, NR, KEGG, SwissProt, and COG databases was conducted to obtain the associated gene name and gene ontology (GO) term accession number, and GO analysis was performed with WEGO software (Ye et al., 2006).

\section{Differential Gene Expression Analysis}

Differential expression analysis between each pair of samples was performed using the DEG-seq R package (Robinson et al., 2010). $P$ values were adjusted using $q$ values (Shannon et al., 2003). The threshold for significantly differential expression was set at a $\mathrm{q}$ value $<0.005$ and $\mid \log 2$ (fold change) $\mid>1$. Based on the hypergeometric distribution model, GO and KEGG ontology enrichment analyses were conducted on the differentially expressed genes (DEGs). GO enrichment analysis of the DEGs was implemented by the GO-seq $\mathrm{R}$ package-based Wallenius noncentral hypergeometric distribution (Young et al., 2010), which can adjust for gene length bias in DEGs. KEGG is a database resource used to understand high-level functions and utilities of biological systems such as the cell, organism, and ecosystem from information at the molecular level, especially large-scale molecular data sets generated by genome sequencing and other high-throughput experimental technologies (http://www. genome.jp/kegg/). We used KOBAS software to test the statistical enrichment of DEGs in KEGG pathways (Mao et al., 2005).

\section{Quantitative Real-Time PCR Validation}

We selected some genes randomly to validate the transcriptome data by using qRT-PCR with gapdh as an internal control. Firststrand CDNA was obtained from the total RNA using random primers and the MMLV reverse transcriptase (Promega, Madison, WI, USA). Primers (listed in Table S1) were designed using Beacon software. The qRT-PCR was performed with SYBR Green PCR Super Mix (Thermo Scientific, Wilmington, DE, USA) and the CFX96 real-time PCR detection system (Bio-Rad, Hercules, CA, USA). PCR was performed in a $10-\mu \mathrm{l}$ reaction volume containing $0.5 \mu \mathrm{l}$ of each primer $(5 \mu \mathrm{M}), 0.5 \mu \mathrm{l}$ cDNA, $5 \mu \mathrm{l}$ SYBR Green Super Mix, and $3.5 \mu \mathrm{ldd} \mathrm{H}_{2} \mathrm{O}$. The PCR cycle was performed as follows: $95^{\circ} \mathrm{C}$ for $7 \mathrm{~min}$, followed by 40 cycles of $95^{\circ} \mathrm{C}$ for $10 \mathrm{~s}, 55^{\circ} \mathrm{C}$ for $15 \mathrm{~s}$, and $72^{\circ} \mathrm{C}$ for $15 \mathrm{~s}$. Three technical replicates and three biological replicates of each sample were run along with the internal control gene. Differences in the expression levels of the WT, MU1 and MU2 fish were assessed after first normalizing expression levels to those of gapdh, followed by log transformation.

\section{RESULTS}

\section{Differences in Chromatophores Among Three Different Phenotypes of $P$. maxillaris}

Chromatophores are mainly responsible for the generation of body color and can be further subdivided based on differences 
in body color. The types and distribution of pigment cells significantly differed among fish exhibiting the different body color phenotypes upon morphological observation. The WT phenotype was much more common than the MU1 and MU2 phenotypes, and WT individuals showed a black and gray body color (Figure 1 A1). The MU1 fish were translucent and showed a silvery-white body color (Figure 1 A2), and the MU2 individuals were completely transparent with clearly visible gill filaments and visceral tissues (Figure 1 A3).

Pigment cell types are easily identified due to their colors and shapes. Three types of pigment cells, i.e., melanophores, xanthophores, and iridophores, were observed in the WT operculum lining, skin, and peritoneum (Figures 1 B1, C1, D1). Nevertheless, in the MU1 fish, melanophores were missing in the

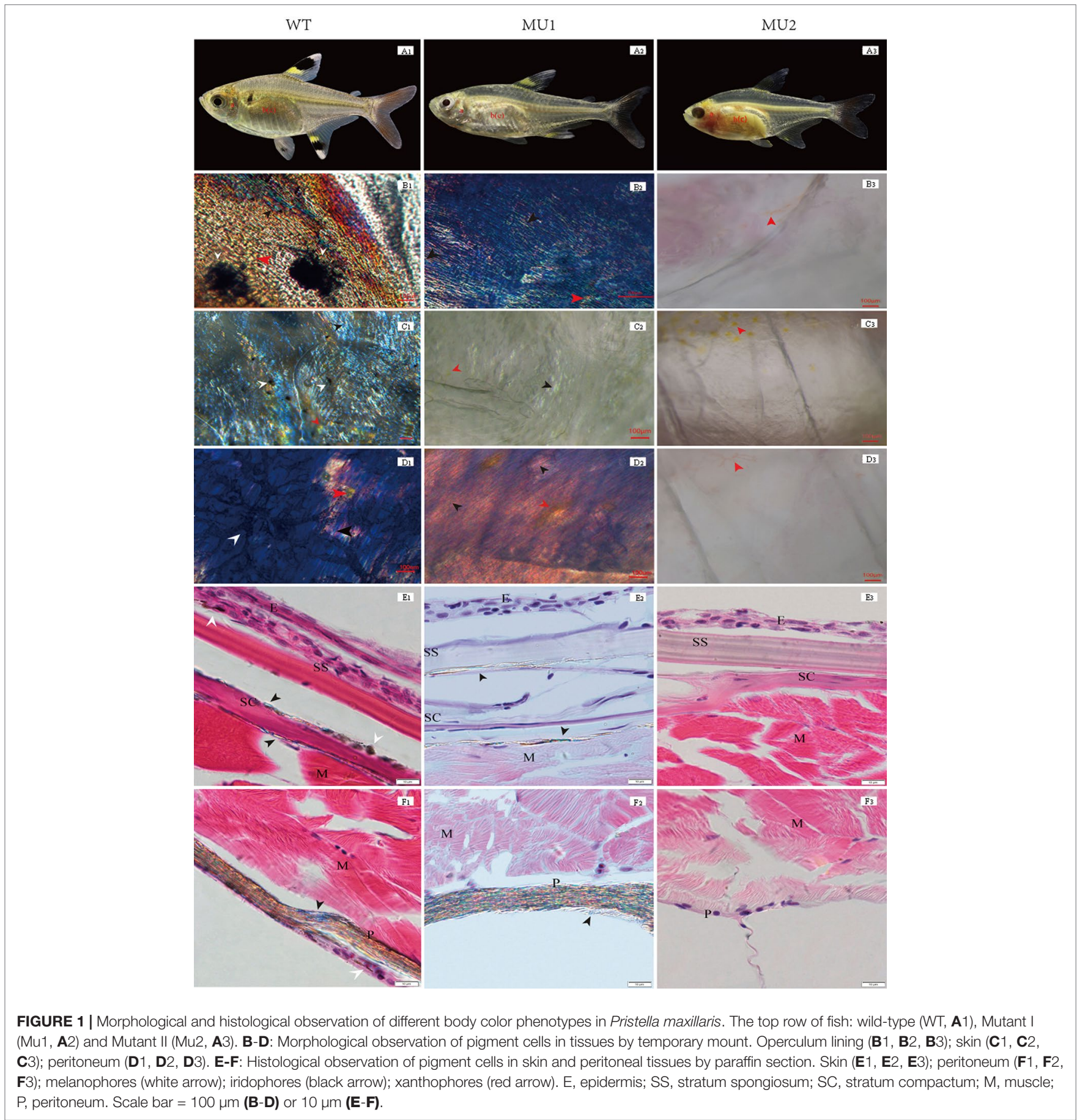


operculum lining, skin, and peritoneal tissues, which contained only two types of pigment cells, iridophores and xanthophores (Figures 1 B2, C2, D2). Without melanophores and iridophores, the MU2 individuals were fully transparent (Figures 1 B3, C3, D3). The histological observation was used to compare the skin and peritoneum of fish exhibiting three different phenotypes of $P$. maxillaris. WT fish had many melanophores and iridophores in their skin and peritoneum (Figures 1 E1, F1). However, MU1 fish had many iridophores in their skin and peritoneum but had no melanophores, which was different from those in WT fish (Figures 1 E2, F2). In addition, melanophores and iridophores were not observed in MU2 fish (Figures 1 E3, F3).

\section{Sequencing and Assembly of the $P$. maxillaris Transcriptome}

To better understand the genetics of the translucent body phenotypes, we conducted a comparative transcriptomic analysis among the three different phenotypes of $P$. maxillaris (WT, MU1, and MU2) using next-generation sequencing. After filtering the raw reads, 72.49 million, 71.70 million, and 74.89 million clean reads were generated from the skin and peritoneal tissues of WT, MU1, and MU2 fish, respectively. The detailed sequencing results are summarized in Table $\mathbf{1 .}$

Transcriptome assemblies obtained from the three different transcriptome libraries were pooled and used to assemble full-length transcripts without reference genomes by Trinity software. After the elimination of redundant transcripts, 166,089 unigenes were acquired that ranged from 201 to 50,278 base pairs (bp) in length with a mean length of 1,293 bp and an N50 of 2,018 bp (Supplementary Table S2). In addition, the size distribution of the transcripts and unigenes is presented in Figure 2A. The unigenes provided the basis for the gene expression analysis in the skin and peritoneal tissues from fish exhibiting the three phenotypes of $P$. maxillaris.

\section{Annotation and Functional Classification}

To identify functional information about the assembled unigenes, all of 166,089 unigenes sequences were used to search against four public databases: the NCBI non-redundant protein (Nr) database, euKaryotic Ortholog Groups (KOG), the Gene Ontology (GO) database, and the Protein family (Pfam) database. The annotation results are demonstrated by a Venn diagram (Figure 2B). Approximately 94,111 (56.61\%), 130,854 (78.78\%), 35,703 (21.49\%), 78,431 (47.22\%), and 77,763 (46.82\%) of the unigenes were identified from the $\mathrm{Nr}, \mathrm{Nt}, \mathrm{KOG}, \mathrm{KEGG}$, and Pfam

TABLE 1 | Summary statistics of transcriptome sequencing for three different phenotypes of Pristella maxillaris.

\begin{tabular}{lccc}
\hline Parameters & WT & MU1 & MU2 \\
\hline Total raw reads & 74349052 & 72769308 & 75931960 \\
Total clean reads & 72492404 & 71695684 & 74893432 \\
Error (\%) & $0.02 \%$ & $0.02 \%$ & $0.02 \%$ \\
Q20 percentage & $97.25 \%$ & $97.62 \%$ & $97.46 \%$ \\
Q30 percentage & $92.66 \%$ & $93.53 \%$ & $93.20 \%$ \\
N percentage & $0.00 \%$ & $0.00 \%$ & $0.00 \%$ \\
GC percentage & $49.17 \%$ & $49.97 \%$ & $49.29 \%$
\end{tabular}

databases, respectively (Supplementary Table S3). Furthermore, $139,108(83.75 \%)$ unigenes were simultaneously annotated in more than one database. Analysis of the BLASTX top-hit species distribution showed that 58,760 (35.20\%) unigenes were similar to the Astyanax mexicanus sequence, 10,476 (6.31\%) were similar to the Danio rerio sequence, $3,037(1.83 \%)$ were similar to the Clupea harengus sequence, and 2,216 (1.33\%) were similar to the Oncorhynchus mykiss sequence (Figure 3).

\section{Recognition of DEGs in Three Different P. maxillaris Phenotypes}

To reveal differences in the chromatophores of the skin and peritoneal tissues in $P$. maxillaris with different phenotypes, we performed a comparative analysis of the three transcriptomes. Based on criteria in which a $\mathrm{q}$ value $<0.005$ and $\mid \log 2$ (fold change) $\mid>1$ indicate a DEG, we identified 3,808 DEGs between MU1 and WT fish, of which 1,698 were upregulated and 2,110 were downregulated. We also identified 4,699 DEGs between MU2 and WT fish, including 1,859 upregulated genes and 2,840 downregulated genes. In addition, 3,109 DEGs were detected between MU1 and MU2 fish, of which 1,661 were upregulated and 1,448 were downregulated (Figure 4).

\section{Functional Enrichment of Differentially Expressed Genes}

By further analysis of GO term enrichment and the KEGG pathways of the DEGs, all the DEGs were classified into different gene ontologies and pathways. After GO annotation, the DEGs between the MU1 and WT, MU2 and WT, and MU1 and MU2 fish were classified into 58 GO terms, 60 GO terms, and $61 \mathrm{GO}$ terms, respectively. Most DEGs were mainly enriched in the following pigmentation-related terms: melanosome, pigment catabolic process, tyrosine biosynthetic process, tRNA (guanine) methyltransferase activity, pigment metabolic process, tyrosine metabolic process, calcium ion transport, purine nucleoside metabolic process, activation of MAPK activity, purine ribonucleotide binding, regulation of Wnt signaling pathway, tricarboxylic acid cycle, and purine-containing compound biosynthetic process. The DEGs were classified into biological processes, cellular components, and molecular functions (as shown in Figure 5). The cellular processes and metabolic processes were the two largest categories within the biological processes; the two largest molecular function categories were binding and catalytic activity; the most abundant categories were cell and intracellular for the cellular components.

The DEGs in the skin and peritoneal tissues in three different phenotypes of $P$. maxillaris were annotated in the KEGG database. The DEGs between the MU1 and WT, MU2 and WT, and MU1 and MU2 fish participated in 20 pathways (Table 2) and were significantly enriched. Among the DEGs between the MU1 and WT fish involved in these 20 pathways, most of the DEGs involved in DNA replication, mismatch repair, nucleotide excision repair, oxidative phosphorylation, and the citrate cycle were downregulated in the MU1 fish compared with their expression in the WT fish (Table 2a). At the same time, some of the DEGs involved in tyrosine metabolism and melanogenesis 


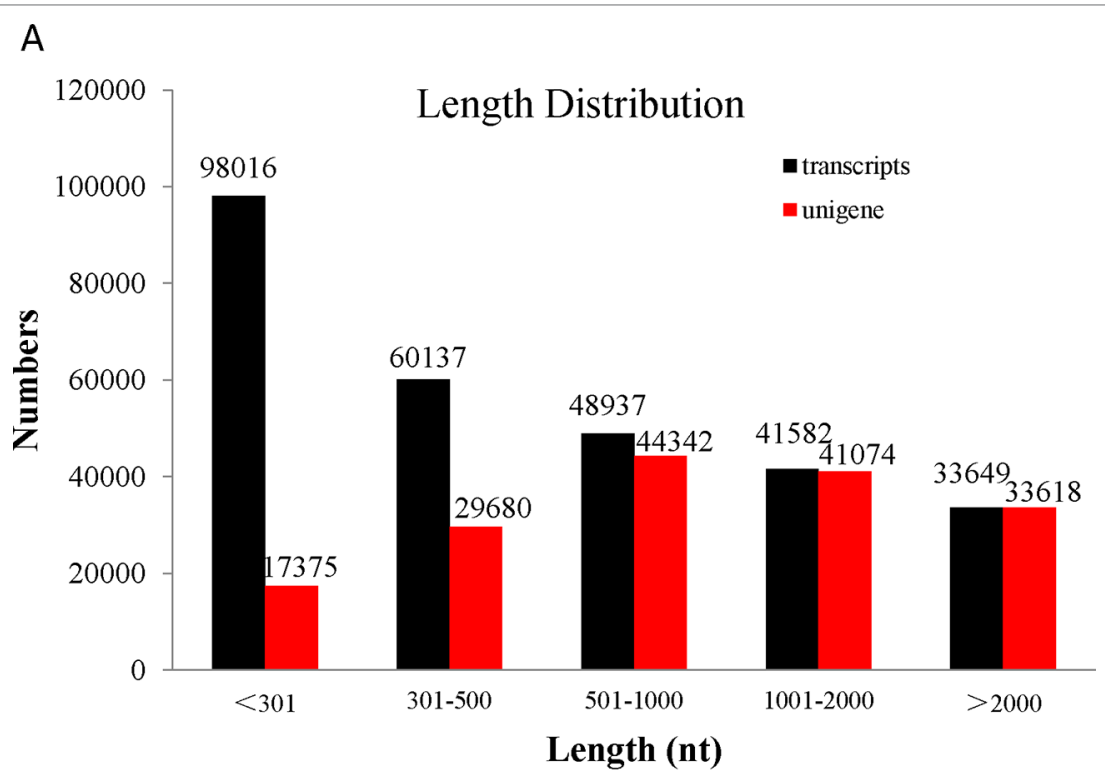

B

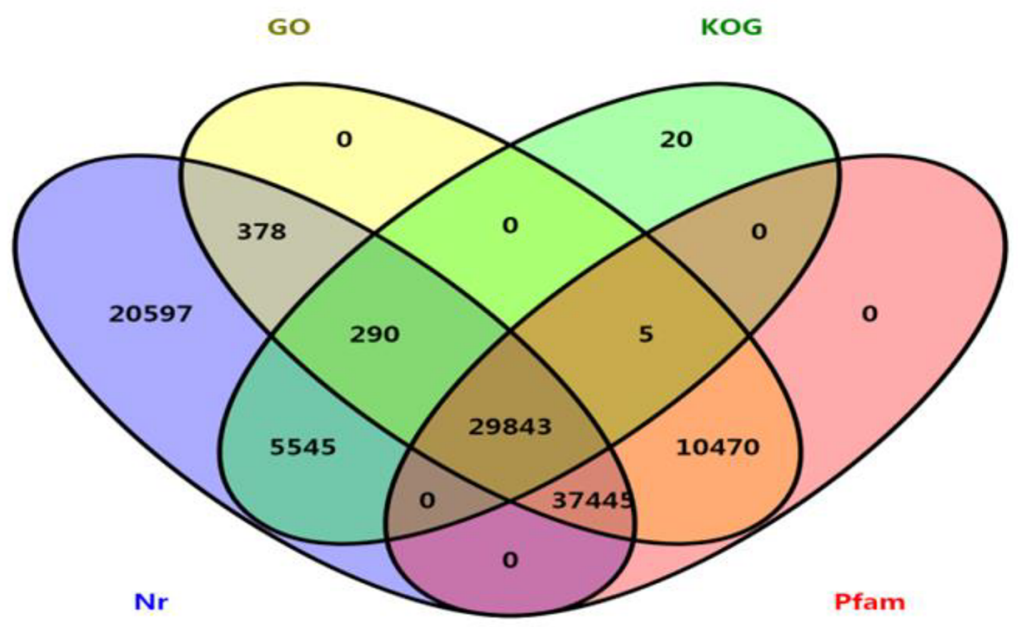

FIGURE 2 | Analysis of the transcriptome data. (A) Length distribution of Pristella maxillaris unigenes and transcripts. (B) Venn diagram of annotation results against $\mathrm{Nr}, \mathrm{KOG}, \mathrm{GO}$, and Pfam databases. The number in each color block indicates the number of unigenes that is annotated by single or multiple databases.

were upregulated in the WT fish compared to their expression in the MU1 fish. The DEGs between the MU2 and WT fish were significantly enriched in some metabolic pathways, including purine metabolism, nucleotide excision repair, the pentose phosphate pathway, glycolysis/gluconeogenesis, mismatch repair, oxidative phosphorylation, tyrosine metabolism, and the melanogenesis pathway (Table $\mathbf{2 b}$ ). Besides, some DEGs involved in purine metabolism, the pentose phosphate pathway, glycolysis/ gluconeogenesis, and tyrosine metabolism were downregulated in the MU2 fish compared with the WT fish. In addition, the ECM receptor interaction, protein digestion and absorption, proteasome, glycolysis/gluconeogenesis, DNA replication, and purine metabolism terms were significantly enriched in most
DEGs between the MU1 and MU2 fish (Table 2c). Meanwhile, some DEGs involved in glycolysis/gluconeogenesis, DNA replication, and purine metabolism were upregulated in the MU1 fish compared with the MU2 fish.

\section{Candidate Genes Related to Chromatophores}

According to zebrafish ensemble database (http://asia.ensembl.org/ Danio rerio/Info/Index), 97 genes were annotated in the pigmentation category. After a BLAST search with the 97 pigmentation-related genes, a total of approximately 40 melanophore- and iridophorerelated genes were detected in the $P$. maxillaris skin and peritoneal 


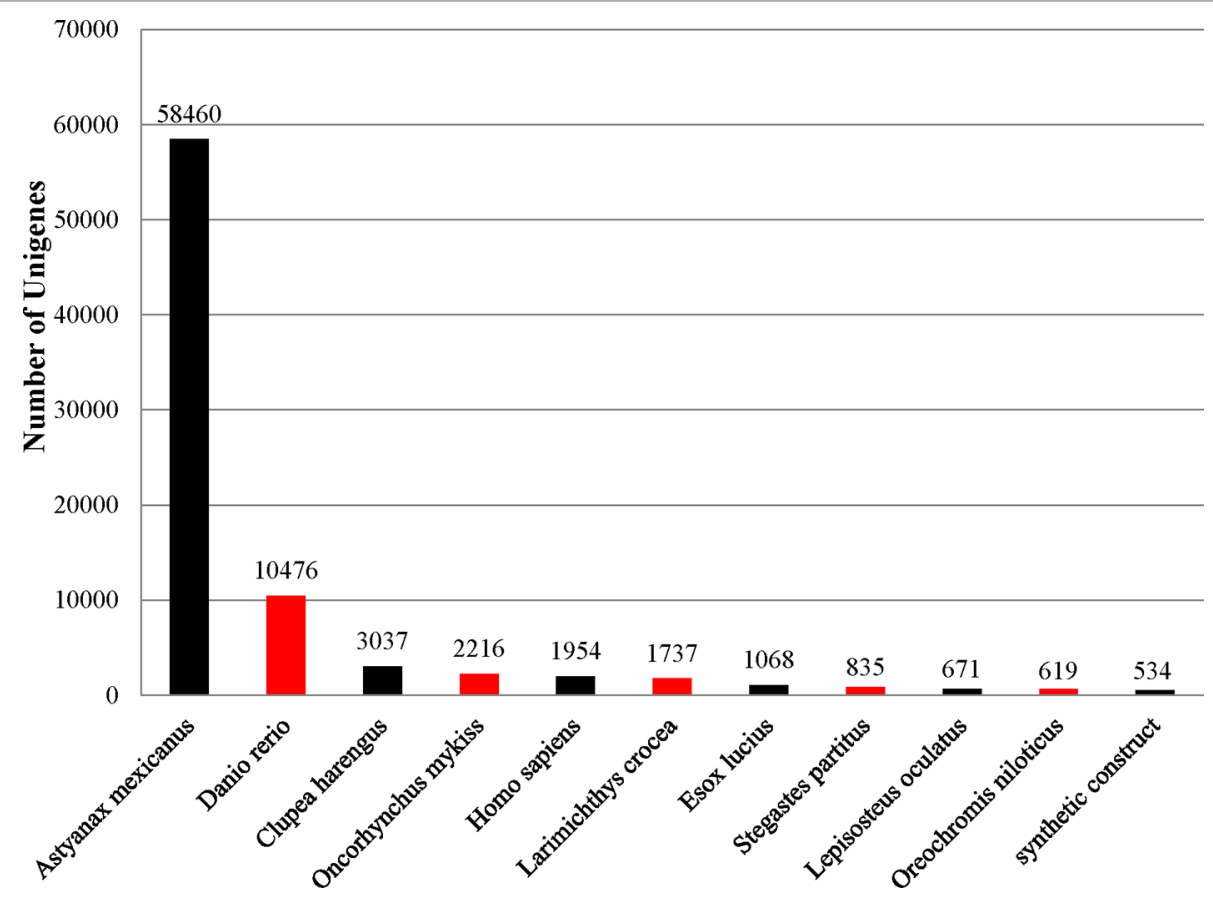

FIGURE 3 | Top-hit species distribution for sequences from Pristella maxillaris submitted BLASTX against the NCBI-Nr database.

tissue. Considering the FPKM (expected number of fragments per kilobase of transcript sequence per millions base pairs sequenced) of these genes, we found 14 genes that enriched the tyrosine metabolism and melanogenesis pathways that were expressed at a significantly higher level in WT fish than in MU1 and MU2 fish and nine genes with significantly higher expression in WT fish (Table 3). Among the DEGs, the protein Wnt-8a (wnt8), frizzled 2 ( $f z d 2$ ), agouti-signaling protein (asip), cyclic AMP-responsive element-binding protein 3-like protein 4 (creb), and dual specificity mitogen-activated protein kinase 2-like (map2k2) were found to be the most highly expressed genes in the WT fish, followed by the alcohol dehydrogenase 6-like (adh6), glutathione S-transferase (gst), guanine nucleotide-binding protein (gnai), and calmodulin-like (cam) genes.

Through GO term and KEGG pathway analyses of the significant DEGs, a total of 26 DEGs were involved in glycolysis/ gluconeogenesis, purine metabolism, and the pentose phosphate pathway, which play an important role in iridophore development. Fifteen of the 26 genes are detailed in Table 4. Interestingly, seven crucial genes were identified in these pathways, including trifunctional purine biosynthetic protein adenosine-3 (gart), hypoxanthine guanine phosphoribosyl transferase (hprt), betaenolase (eno), nucleoside diphosphate kinase-like (ndk), guanylate kinase isoform X1 (guk1a), phosphoglycerate mutase 1-like (pgam1), and 6-phosphofructokinase, muscle type ( $p f k a)$, which were significantly upregulated in the WT and MU1 fish but significantly downregulated in the MU2 fish ( $p$ value $\leq 0.005$ ). In contrast, the expression of the bifunctional purine biosynthesis protein (pur9) and L-lactate dehydrogenase B-A chain (ldh) genes did not differ among the three different translucent body phenotypes.
The GO term enrichment and KEGG pathway analyses of the DEGs identified candidate genes that could regulate melanophore or iridophore development. We also analyzed the mRNA expression of 17 candidate genes (Figure 6). Some genes, such as $w n t 8, f z d 2$, map $2 k 2, c a m, c r e b$, and gst, were significantly upregulated in WT fish compared with the MU1 and MU2 fish. However, the mRNA expression levels of some iridophore-related genes ( $p f k a$, eno, gart, and $h p r t$ ) were markedly lower in the MU2 fish than in the WT and MU1 fish.

\section{Confirmation of DEGs Identified with RNA- Seq by Quantitative Real-Time PCR}

To test the DEGs identified by comparative transcriptomic analysis, we selected nine genes from the three comparative groups and the gapdh gene for qRT-PCR confirmation. The quantitative real-time PCR (qRT-PCR) expression patterns of 6 of 9 randomly selected DEGs that were related to pigment biosynthesis agreed with the results from RNA-Seq analysis, except for the $p k$ (pyruvate kinase), cam, and transketolase-like protein $2(t k t l)$ genes (Figure 7). Therefore, the expression patterns of the selected genes determined by qRT-PCR were nearly in accordance with the RNA-Seq data. Combining the qRT-PCR and RNA-Seq results, we found that melanin-related genes were more highly expressed in the skin and peritoneal tissues of WT fish than in mutant fish, whereas guanine-related genes were more highly expressed in the WT and MU1 fish than in the MU2 fish. However, the melaninrelated genes and guanine-related genes were expressed at lower levels in MU2 fish compared with the WT and MU1 fish. 


\section{A}

MU1vsWT

MU2vsWT

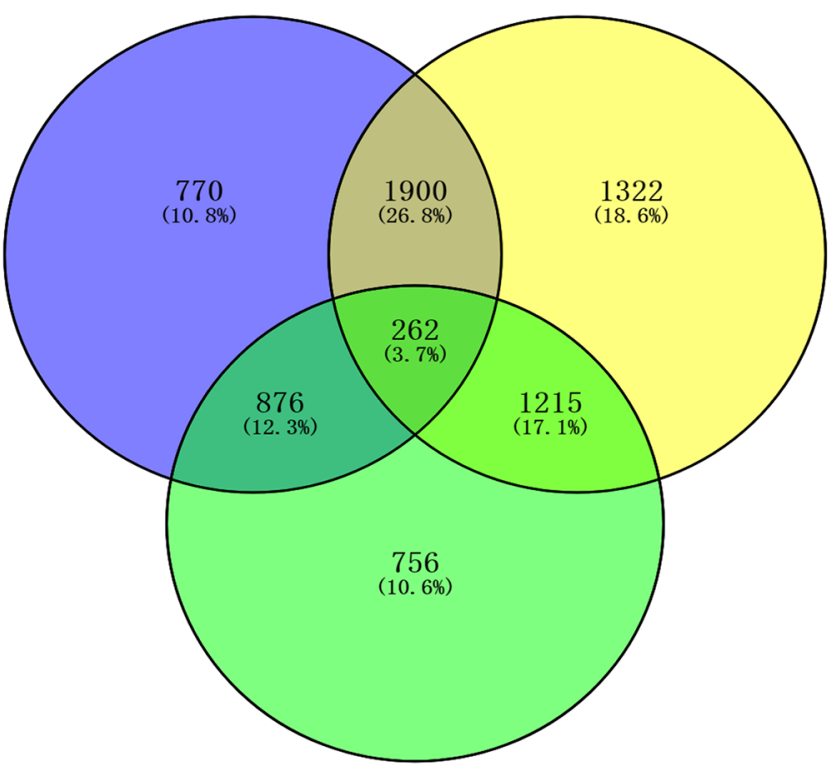

MU1vsMU2

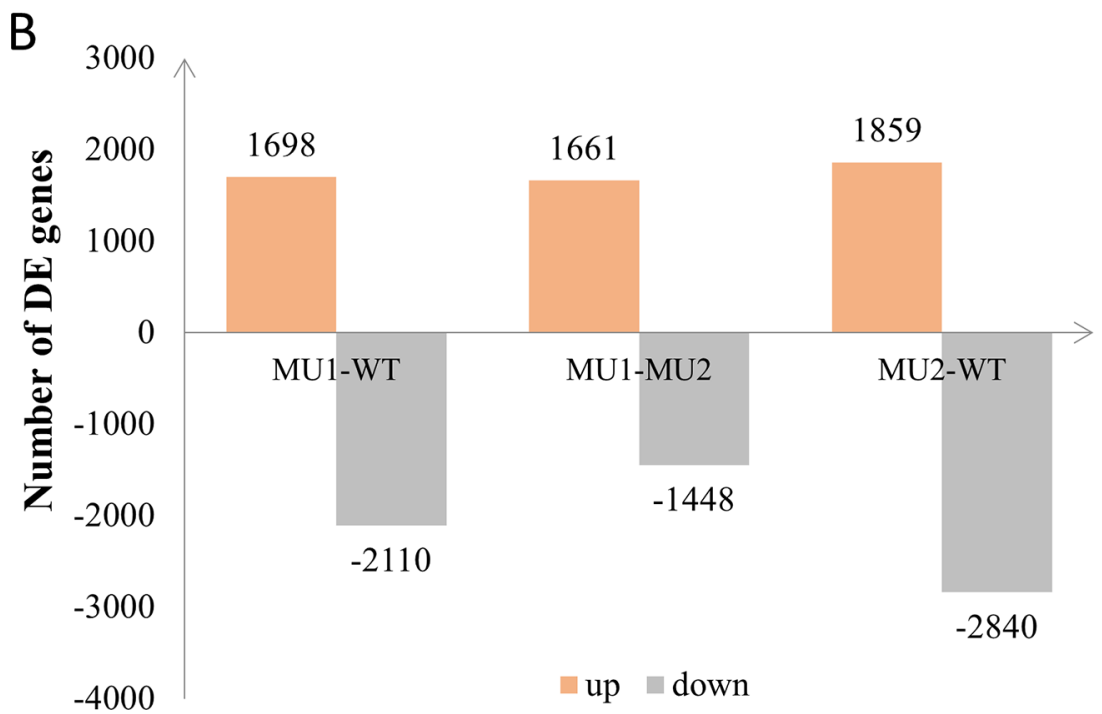

FIGURE $\mathbf{4}$ | Differentially expressed genes (DEGs) in the three different phenotypes of Pristella maxillaris by Venn diagram (A) or bar chart (B). The number of DEGs identified in each library contrast applying a threshold of the ratio change and a $p$ value of $<0.05$. The light red/gray column represents genes up-/down-regulated in different color P. maxillaris.

\section{DISCUSSION}

Animal coloration plays an important role in ecological interactions, species recognition, and even protecting the organism from ultraviolet radiation damage (Muske and Fernald, 1987; Lowe and Goodman-Lowe, 1996; Fujimura et al., 2009; Sefc et al., 2014). Diverse body coloration is mainly controlled by the development and location of pigment cells. The variety and number of pigment cells affect animal body transparency (Nilsson Skold et al., 2010; Croucher et al., 2013; Nilsson Skold et al., 2013). In this study, we observed the different morphologies of transparent body parts from three different phenotypes of $P$. maxillaris, and revealed significant differences in the types and distributions of pigment cells by 


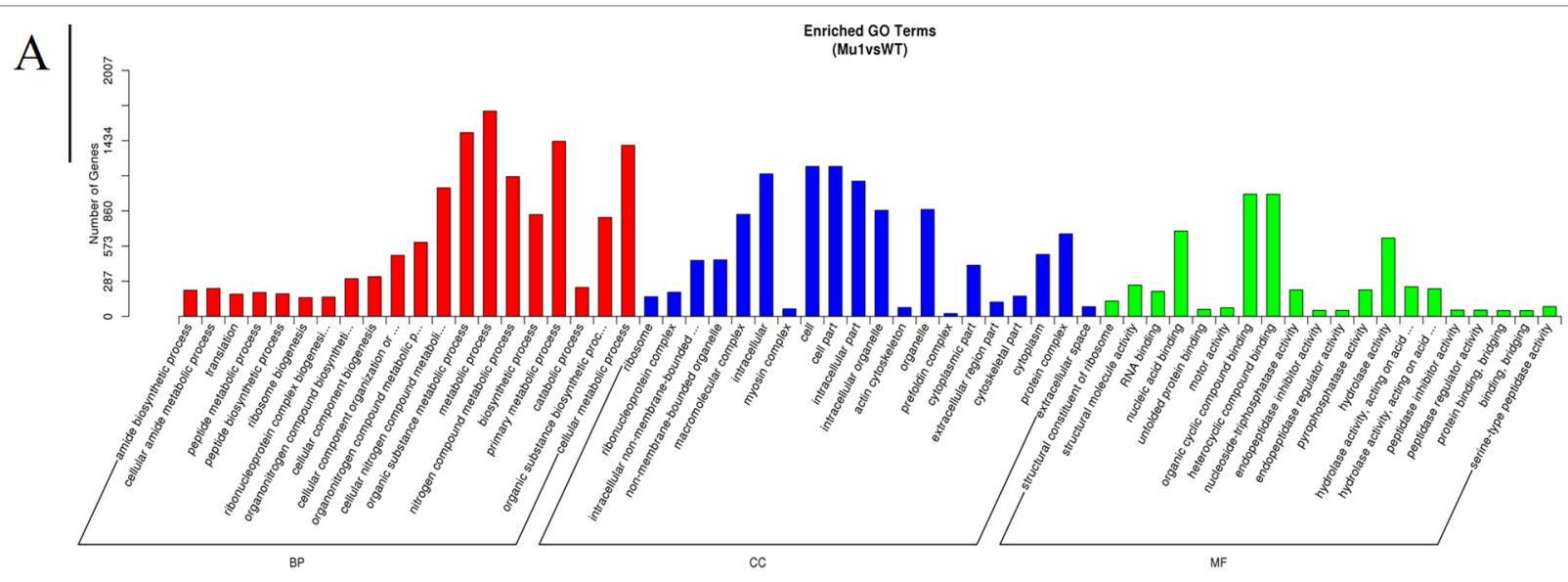

B

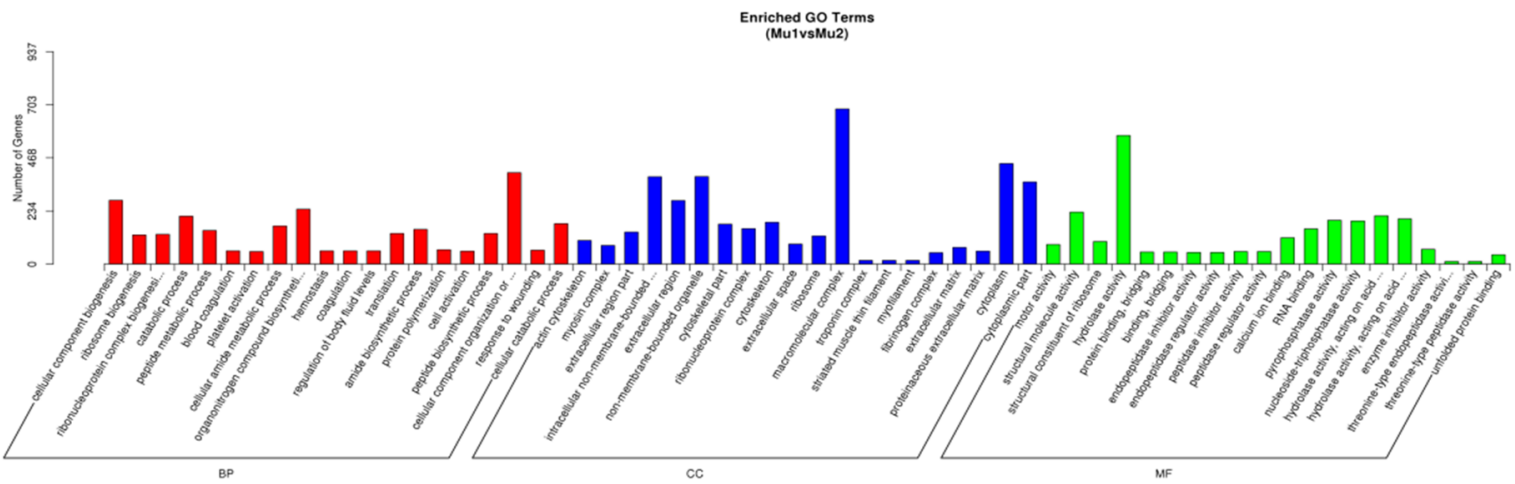

C Enriched Go Terms
(Mu2vsWT)

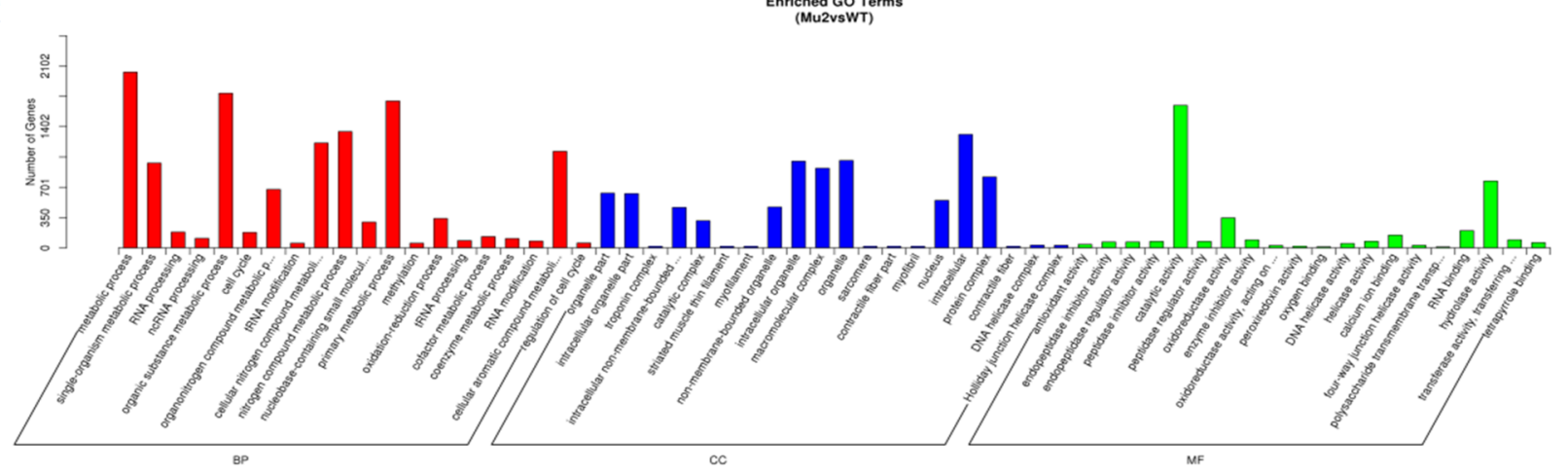

FIGURE 5 | Gene Ontology (GO) functional classification of DEGs in MU1 vs. WT (A), MU2 vs. WT (B), and MU1 vs. MU2 (C).

microscopic observation as performed in other literatures (Kelsh, 2004; Darias et al., 2013; Zhang et al., 2015; Zhang et al., 2017). Moreover, we also found that changes in the type and number of pigment cells led to different phenotypes and increased the transparency of the $P$. maxillaris body. Extensive research has been performed on this topic, and many species of fish have been shown to change their internal color due to responsive peritoneal chromatophores, in which the degree of this response was correlated with the level of body transparency (Parichy, 2007; Nilsson Skold et al., 2010). Meanwhile, Krauss indicated that the inner organs were observed through the skin due to the loss of iridophores (Krauss et al., 2013). Our results suggested that the loss of melanophores and iridophores resulted in changing of body color from gray to transparent during $P$. maxillaris reproduction. In the study, xanthophores were not observed in the different phenotypes fish by histological 
TABLE 2 | Kyoto Encyclopedia of Genes and Genomes (KEGG) functional analysis of DEGs in MU1 vs. WT (a), MU2 vs. WT (b), and MU1 vs. MU2 (c).

\begin{tabular}{|c|c|c|c|}
\hline Pathway (MU1vsWT, a) & DEGs*with Pathway & q-Value & Pathway ID \\
\hline Ribosome & 109 & 1.69E-31 & ko03010 \\
\hline DNA replication & 20 & 6.13E-05 & ko03030 \\
\hline Mismatch repair & 12 & 0.003566778 & ko03430 \\
\hline Systemic lupus erythematosus & 38 & $1.66 \mathrm{E}-10$ & ko05322 \\
\hline Nucleotide excision repair & 19 & $4.52 \mathrm{E}-05$ & ko03420 \\
\hline Staphylococcus aureus infection & 24 & 0.000216615 & ko05150 \\
\hline Glutathione metabolism & 20 & 0.00019 & ko00480 \\
\hline RNA degradation & 28 & 2.77E-05 & ko03018 \\
\hline Pyrimidine metabolism & 42 & 4.45E-07 & ko00240 \\
\hline Legionellosis & 23 & 0.00018 & ko05134 \\
\hline Antigen processing and presentation & 32 & 3.81E-05 & ko04612 \\
\hline Cell cycle & 45 & 2.10E-06 & ko04110 \\
\hline Oxidative phosphorylation & 38 & 1.26E-05 & ko00190 \\
\hline Parkinson's disease & 44 & $5.55 E-06$ & ko05012 \\
\hline RNA transport & 59 & 7.41E-07 & ko03013 \\
\hline Purine metabolism & 51 & $6.54 \mathrm{E}-05$ & ko00230 \\
\hline MAPK signaling pathway & 30 & 0.997786982 & ko04010 \\
\hline cAMP signaling pathway & 22 & 0.996297986 & ko04024 \\
\hline Melanogenesis & 44 & 0.910326122 & ko04916 \\
\hline Tyrosine metabolism & 4 & 0.50194862 & ko00350 \\
\hline Pathway (MU2vsWT, b) & DEGs*with Pathway & q-Value & Pathway ID \\
\hline Pyrimidine metabolism & 65 & $1.25 E-11$ & ko00240 \\
\hline DNA replication & 33 & $6.91 \mathrm{E}-11$ & ko03030 \\
\hline Cell cycle & 69 & $6.91 \mathrm{E}-11$ & ko04110 \\
\hline Systemic lupus erythematosus & 44 & 2.56E-09 & ko05322 \\
\hline Purine metabolism & 71 & $1.92 \mathrm{E}-06$ & ko00320 \\
\hline Nucleotide excision repair & 27 & 4.80E-06 & ko03420 \\
\hline Spliceosome & 59 & 8.56E-05 & ko03040 \\
\hline Pentose phosphate pathway & 19 & 0.000103465 & ko00030 \\
\hline Mismatch repair & 16 & 0.000320844 & ko03430 \\
\hline RNA polymerase & 17 & 0.000522379 & ko03020 \\
\hline Staphylococcus aureus infection & 26 & 0.000547331 & ko05150 \\
\hline RNA degradation & 32 & 0.000635092 & ko03018 \\
\hline Glycine, serine and threonine metabolism & 18 & 0.001075308 & ko00260 \\
\hline Cardiac muscle contraction & 43 & 0.001075308 & ko04260 \\
\hline ECM-receptor interaction & 43 & 0.002053638 & ko04512 \\
\hline Parkinson's disease & 46 & 0.002072794 & ko05012 \\
\hline Oxidative phosphorylation & 39 & 0.004989829 & ko00190 \\
\hline Glycolysis / Gluconeogenesis & 26 & 0.008887468 & ko00010 \\
\hline Tyrosine metabolism & 8 & 0.090654 & ko00350 \\
\hline Melanogenesis & 22 & 0.822218 & ko04916 \\
\hline Pathway (MU1vsMU2, c) & DEGs*with Pathway & q-Value & Pathway ID \\
\hline Ribosome & 89 & $2.75 E-25$ & ko03010 \\
\hline ECM-receptor interaction & 61 & $2.63 E-11$ & ko04512 \\
\hline Protein digestion and absorption & 75 & $5.32 \mathrm{E}-18$ & ko04974 \\
\hline Tight junction & 93 & $8.50 E-13$ & ko04530 \\
\hline Pathogenic Escherichia coli infection & 42 & $1.08 \mathrm{E}-07$ & ko05130 \\
\hline Proteasome & 20 & $2.32 \mathrm{E}-07$ & ko03050 \\
\hline Glycolysis / Gluconeogenesis & 29 & 2.90E-07 & ko00010 \\
\hline Hypertrophic cardiomyopathy (HCM) & 44 & 4.50E-06 & ko05410 \\
\hline DNA replication & 16 & 4.74E-05 & ko03030 \\
\hline PI3K-Akt signaling pathway & 89 & 0.000509 & ko04151 \\
\hline Focal adhesion & 87 & $1.08 \mathrm{E}-05$ & ko04510 \\
\hline Dilated cardiomyopathy (DCM) & 44 & $1.20 \mathrm{E}-05$ & ko05414 \\
\hline Systemic lupus erythematosus & 25 & 3.36E-05 & ko05322 \\
\hline Metabolism of xenobiotics by cytochrome P450 & 12 & 0.047531 & ko00980 \\
\hline Purine metabolism & 33 & 0.080042 & ko00230 \\
\hline Pentose pphosphate athway & 11 & 0.001984 & ko00030 \\
\hline Spliceosome & 43 & $6.88 \mathrm{E}-05$ & ko03040 \\
\hline Cardiac muscle contraction & 33 & 0.000210 & ko04260 \\
\hline
\end{tabular}


TABLE 2 | Continued

\begin{tabular}{lcc}
\hline Pathway (MU1vsWT, a) & DEGs*with Pathway & Pathway ID \\
\hline Antigen processing and presentation & 27 & 0.000417 \\
Arginine biosynthesis & 13 & $k 004612$ \\
\hline
\end{tabular}

*DEGs, differentially expressed genes, which was identified by the DEGseq package. DEGs between the two samples were selected with the following filter criteria: log2 transcript abundance ratio $\geq 1$ and FDR (false discovery ratio) $\leq 0.001$.

TABLE 3 | KEGG pathway analysis of positively selected genes involved in melanophores in P. maxillaris.

\begin{tabular}{|c|c|c|c|}
\hline Gene ID & Gene name & Discription & KEGG pathway \\
\hline Cluster-7922.70780 & adh6 & alcohol dehydrogenase 6-like & Tyrosine metabolism \\
\hline Cluster-7922.15576 & gst & glutathione S-transferase & Drug metabolism - cytochrome P450 \\
\hline Cluster-7922.27281 & wnt8 & protein Wnt-8a & Wnt/ $\beta$-catenin signaling pathway \\
\hline Cluster-7922.40920 & $f z d 2$ & frizzled 2 & Melanogenesis \\
\hline Cluster-7922.92050 & asip & agouti-signaling protein & Melanogenesis \\
\hline Cluster-7922.45484 & creb3 & cyclic AMP-responsive element-binding protein 3-like protein 4, & Melanogenesis \\
\hline Cluster-7922.33657 & map2k2 & dual specificity mitogen-activated protein kinase kinase 2-like & MAPK signaling pathway \\
\hline Cluster-7922.68121 & gnai & guanine nucleotide-binding protein $\mathrm{G}(\mathrm{o})$ subunit alpha isoform $\mathrm{X}_{1}$ & Melanogenesis \\
\hline Cluster-7922.65792 & cam & calmodulin-like & Melanogenesis \\
\hline Cluster-7922.58712 & bad & bcl2 antagonist of cell death-like isoform $X_{1}$ & Melanoma \\
\hline Cluster-7922.100710 & $c d k 4$ & cyclin-dependent kinase 4 & Melanoma \\
\hline Cluster-7922.81890 & $d v / 2$ & segment polarity protein dishevelled homolog & Melanogenesis \\
\hline Cluster-7922.62453 & kras & GTPase KRas isoform X1 & Melanogenesis \\
\hline Cluster-7922.78643 & camk2 & calcium/calmodulin-dependent protein kinase type II & Melanogenesis \\
\hline
\end{tabular}

TABLE 4 | KEGG pathway analysis of positively selected genes involved in iridophores metabolism in P. maxillaris.

\begin{tabular}{|c|c|c|c|}
\hline Gene ID & Gene name & Discription & KEGG Pathway \\
\hline Cluster-7922.60112 & ldh & L-lactate dehydrogenase B-A chain & Glycolytic pathway \\
\hline Cluster-7922.64440 & pk & pyruvate kinase PKM isoform X1 & Purine metabolism \\
\hline Cluster-7922.57158 & eno & beta-enolase & Glycolysis / Gluconeogenesis \\
\hline Cluster-7922.63283 & aldo & fructose-bisphosphate aldolase C-B-like & Glycolysis / Gluconeogenesis \\
\hline Cluster-7922.61593 & pur9 & bifunctional purine biosynthesis protein & Purine metabolism \\
\hline Cluster-7922.45519 & hprt & hypoxanthine-guanine phosphoribosyltransferase & Purine metabolism \\
\hline Cluster-7922.59115 & pgam1 & phosphoglycerate mutase 1-like & Glycolysis / Gluconeogenesis \\
\hline Cluster-7922.51649 & guk1a & guanylate kinase isoform $X_{1}$ & Purine metabolism \\
\hline Cluster-7922.49116 & pfka & 6-phosphofructokinase, muscle type & pentose phosphate pathway \\
\hline Cluster-7922.79755 & tktl & transketolase-like protein 2 & pentose phosphate pathway \\
\hline Cluster-7922.64418 & taldo & transaldolase & pentose phosphate pathway \\
\hline Cluster-7922.34530 & pgd & 6-phosphogluconate dehydrogenase, decarboxylating-like & pentose phosphate pathway \\
\hline Cluster-7922.52874 & impdh2 & monophosphate dehydrogenase 2 & Purine metabolism \\
\hline Cluster-7922.21114 & $n d k$ & nucleoside diphosphate kinase-like isoform $\mathrm{X}_{1}$ & Purine metabolism \\
\hline Cluster-7922.62678 & gart & trifunctional purine biosynthetic protein adenosine-3 & Purine metabolism \\
\hline
\end{tabular}

method. The reason may be that the inclusions of xanthophores are fat-soluble carotenoids and water-soluble dinidine (Hirata et al., 2003), which are easy to dissolve during dehydration and repeated washing. In future studies, we will try to use the other method to observe the xanthophores.

Genetic factors, which are the major determinants of animal body color, influence the production and distribution of pigment cells. In recent years, the mechanism of body color formation in fish has received attentions such as transcriptome analyses of model or economic fish including zebrafish (Irion et al., 2016), crucian carp (Zhang et al., 2017), and the common carp (Wang et al., 2014). In this study, we used
Illumina sequencing technology to examine the skin and peritoneal tissues from fish exhibiting three phenotypes of $P$. maxillaris at the transcriptome level and found many DEGs associated with pigmentation. The identified DEGs among the three phenotypes could help us understand the molecular mechanism and provide valuable genetic information to explore pigmentation in the future.

The GO enrichment analysis of the DEGs revealed that variations in pigmentation are related to cellular components and biological processes. Most of the clustered groups of DEGs were consistent with those identified in previous works with fish such as zebrafish (Higdon et al., 2013), Midas cichlids 


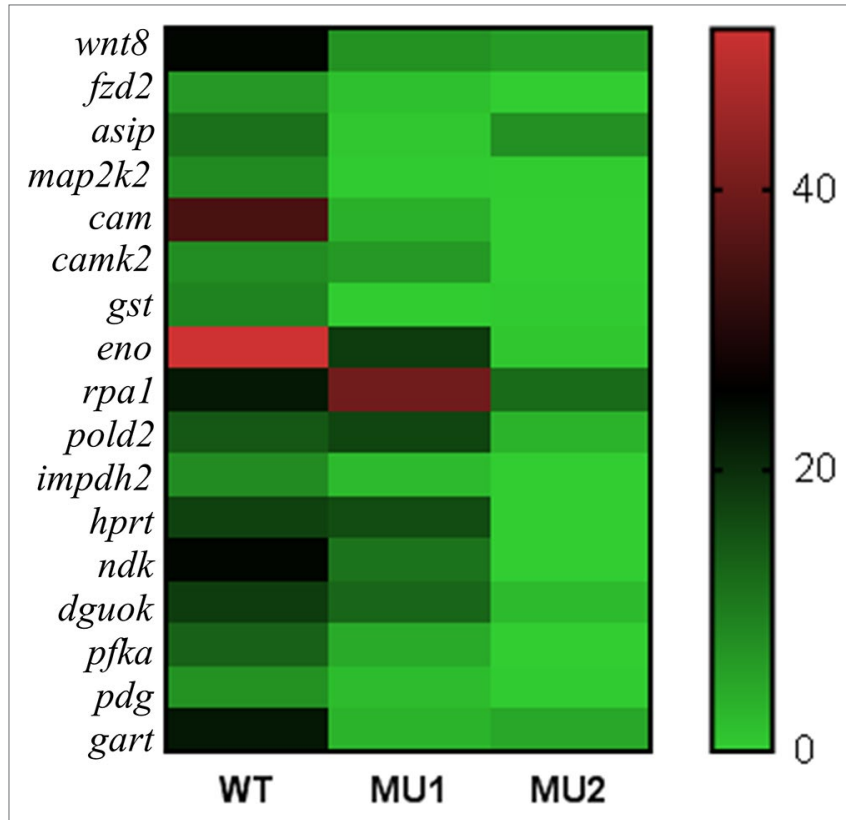

FIGURE 6 | Heat map showing the expressions of selected DEGs in three body color phenotypes of $P$. maxillaris.

(Henning et al., 2013), crucian carp (Zhang et al., 2017), and common carp (Jiang et al., 2014; Li et al., 2015). Interestingly, we found that most of the genes downregulated in the MU2 fish compared to their expression in the WT fish were enriched in GO terms related to the pigment metabolic process, including the tyrosine metabolic process, the activation of MAPK activity, tyrosine 3-monooxygenase activity, the pigment catabolic process, the purine-containing compound biosynthetic process, tRNA (guanine) methyltransferase activity, and the purine nucleobase biosynthetic process. In addition, most of the genes downregulated in the MU1 fish compared with their expression in the WT fish were enriched in the tyrosine metabolic process, activation of MAPK activity, tyrosine 3-monooxygenase activity, and pigment biosynthetic process GO terms.

The KEGG pathway analysis showed that some DEGs were associated with pigmentation-related pathways. In our study, some DEGs between the MU1 and WT fish were enriched in the tyrosine metabolism, melanogenesis, cAMP signaling, and Wnt or MAPK signaling pathways. Both the cAMP and MAPK signaling pathways are involved in melanophore development in vertebrates (Zhang et al., 2015; Zhang et al., 2017). The DEGs in $P$. maxillaris were likely involved in melanin synthesis. Meanwhile, we found that some DEGs between the MU2 and WT fish were enriched in the glycolysis/gluconeogenesis, purine metabolism, and pentose phosphate pathway terms. The identification of genes enriched in these pigmentation-related terms and pathways are informative, and these genes are worth further study.

In this study, comparing known pigmentation genes with identified genes by the current transcriptome data, we found many of the pigmentation genes and pathways in P. maxillaris. The putative genes and pathways involved in the three body transparency phenotypes that are related to the pigmentation process are shown in Figure 8. We found that the mRNA expression levels of $w n t 8, f z d 2, m a p 2 k 2, c r e b$, asip, and cam were downregulated in the skin and peritoneal tissues of MU2 fish compared to the WT fish. Several studies have reported that the Wnt signaling pathway participates in the synthesis of melanogenesis in teleost fishes, as well as in mammals (Fujimura et al., 2009; Xing et al., 2011; Zhang et al., 2015). Wnt8, a noncanonical Wnt protein family gene, was found in the matrix and precortical cells in the hair follicles of mice (Yamaguchi et al., 1999; Croucher et al., 2013). Interestingly, wnt8 was expressed at lower levels in the MU1 and MU2 fish than in the WT fish. The wnt 8 can bind with $f z d 2$ to promote the production of guanine-binding protein $(\mathrm{Go} / \mathrm{Gq})$, which in turn promotes the expression of $\beta$-catenin, thereby inducing the expression of mitf (melanocyte inducing transcription factor). The mitf is a key regulatory gene in the melanophore lineage (Levy et al., 2006; Zeng et al., 2015). Some transcription factors, such as $\beta$-catenin and sox10 (SRY-box containing gene 10), have been reported to act on the promoter region of mitf, which promotes the expression of mitf (Sakai et al., 1997; Zhang et al., 2017). In addition, mitf directly regulates the expression of multiple genes (tyr, tyrp1, and $d c t$ [dopachrome tautomerase]) that are necessary for the survival and proliferation of melanophores (Opdecamp et al., 1997; Cheli et al., 2010) and are responsible for the synthesis of melanin (Li et al., 2012). These results show that $w n t 8, f z d 2$, and $\beta$-catenin might play important roles in the body transparency phenotypes of $P$. maxillaris.

We also found that the dual specificity map $2 k 2$ and cam genes were significantly upregulated in the WT fish compared to the MU1 and MU2 fish. Cam is activated by cytoplasmic $\mathrm{Ca}^{2+}$, which is released from the endoplasmic reticulum to assist protein kinase C (Ma et al., 2014). Additionally, protein kinase $\mathrm{C}$ can expand the promotion of melanin synthesis by protein kinase A by upregulating the mitf gene (Park et al., 2006). Another gene, map2k2, encodes an important enzyme in the MAPK signaling pathway that can activate mitf, increase mitf expression, and then stimulate the synthesis of melanin (Levy et al., 2006). In addition, the mRNA expression level of asip decreased as the $P$. maxillaris body color changed from gray to transparent. Asip was an endogenous antagonist of alphamelanocyte stimulating hormone ( $\alpha-\mathrm{MSH})$. The $\alpha-\mathrm{MSH}$ causes an increase in tyrosinase activity, and $\alpha-\mathrm{MSH}$ could activate the melanocortin 1 receptor $(m c 1 r)$, a key gene in melanogenesis in animals, resulting in increased cAMP levels. Consequently, the melanin biosynthesis process is triggered (Voisey et al., 2001; Henning et al., 2010; Zhang et al., 2017). On the contrary, Asip can block melanin synthesis by competing with a-MSH in binding to the mc1r gene (Sakai et al., 1997; Zhang et al., 2017). Histological assessment revealed that MU1 and MU2 do not possess melanophores. In addition, the results revealed that asip might not inhibit melanin synthesis in MU1 and MU2 fish. We also found that the creb gene was significantly upregulated in the WT fish compared to its expression in MU1 and MU2 fish. It has been reported that $m c 1 r$ activates the $c r e b$, and its cascade involves the upregulation of the expression of mitf, which binds and activates melanogenic gene promoters to increase their expression, resulting in increased melanin synthesis (Busca 

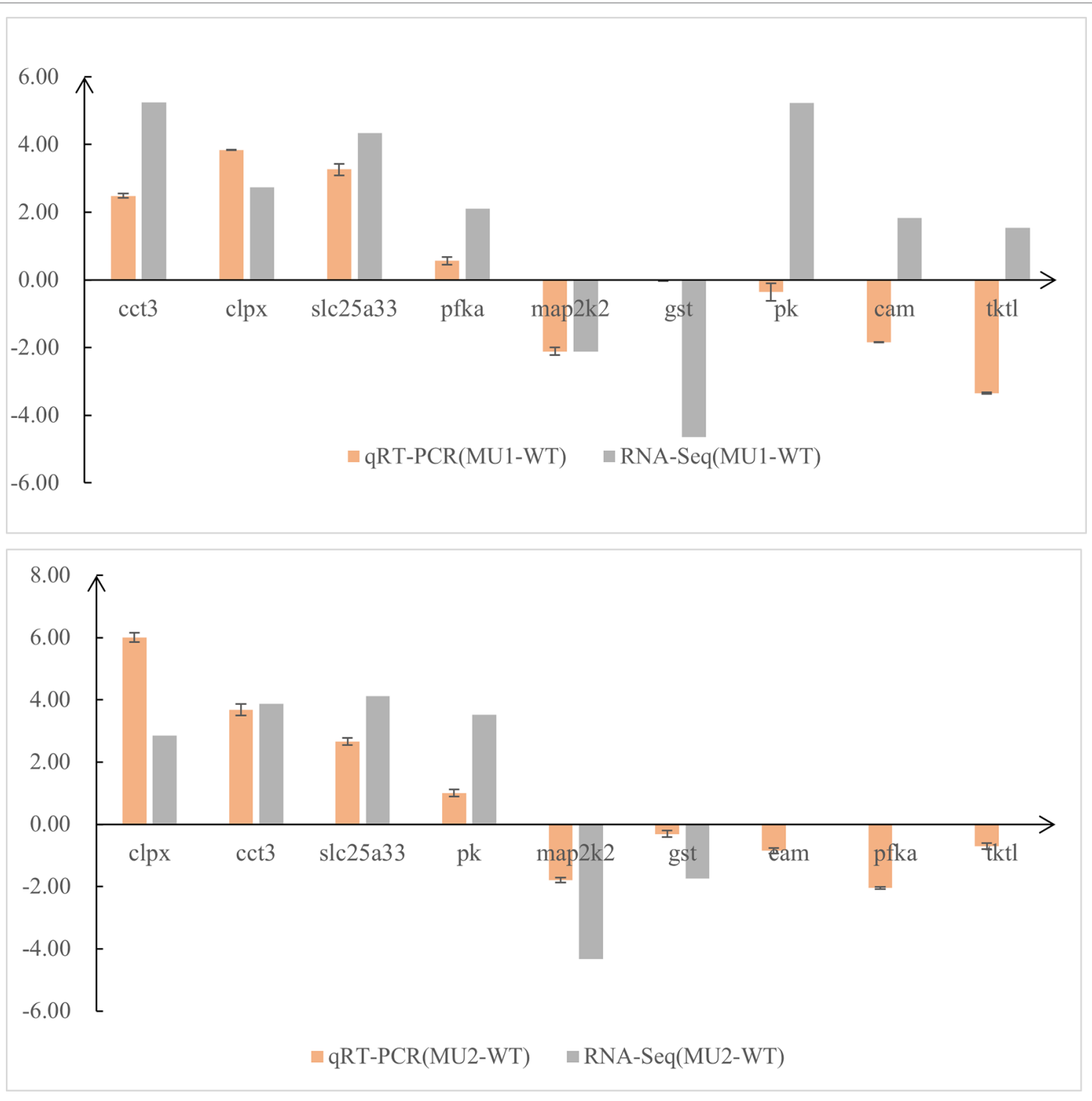

FIGURE 7 | Comparison of gene expression patterns obtained using RNA-Seq and qRT-PCR. Log-fold changes are expressed as the ratio of gene expression after normalization to gapdh.

and Ballotti, 2000). Therefore, it was again confirmed that creb might play a key role in melanin production.

We also discovered some DEGs between group of MU2 and WT and group of MU1 and MU2 fishes involved in purine metabolism, glycolysis/gluconeogenesis, the citric acid cycle, and the pentose phosphate pathway such as the $\beta$-eno, gart, aldo (fructose-bisphosphate aldolase C-B-like), ldh, and hprt genes. These genes exhibited significantly lower expression in MU2 fish compared to the WT and MU1 fish, followed by guk1a and $p f k a$, which implied the participation of these pathways in body coloration in MU2 fish. Fish skin and other tissues contained stacks of guanine plates in iridophores (Hirata et al., 2003; Hirata et al., 2005; Failde et al., 2014), and glycolysis and the citrate cycle pathway were found to be key participants in extensive guanine synthesis (Higdon et al., 2013; Irion et al., 2016). Combined with our microscopic observations, many iridophores were observed in the WT and MU1 fish, but fish with the MU2 mutation did not harbor iridophores. Thus, the increased expression of genes within these pathways might be in accordance with the increased requirement of guanine for the reflective iridophore pigment in the skin and peritoneal tissues of WT and MU1 fish.

In the purine metabolism pathway, the gart and phosphoribo sylaminoimidazolesuccinocarboxamide synthase (paics) genes combine into a complex that is involved in the synthesis of inosine monophosphate, a precursor of the purine nucleotides adenosine monophosphate and guanosine monophosphate. Some studies have shown that guanosine monophosphate 


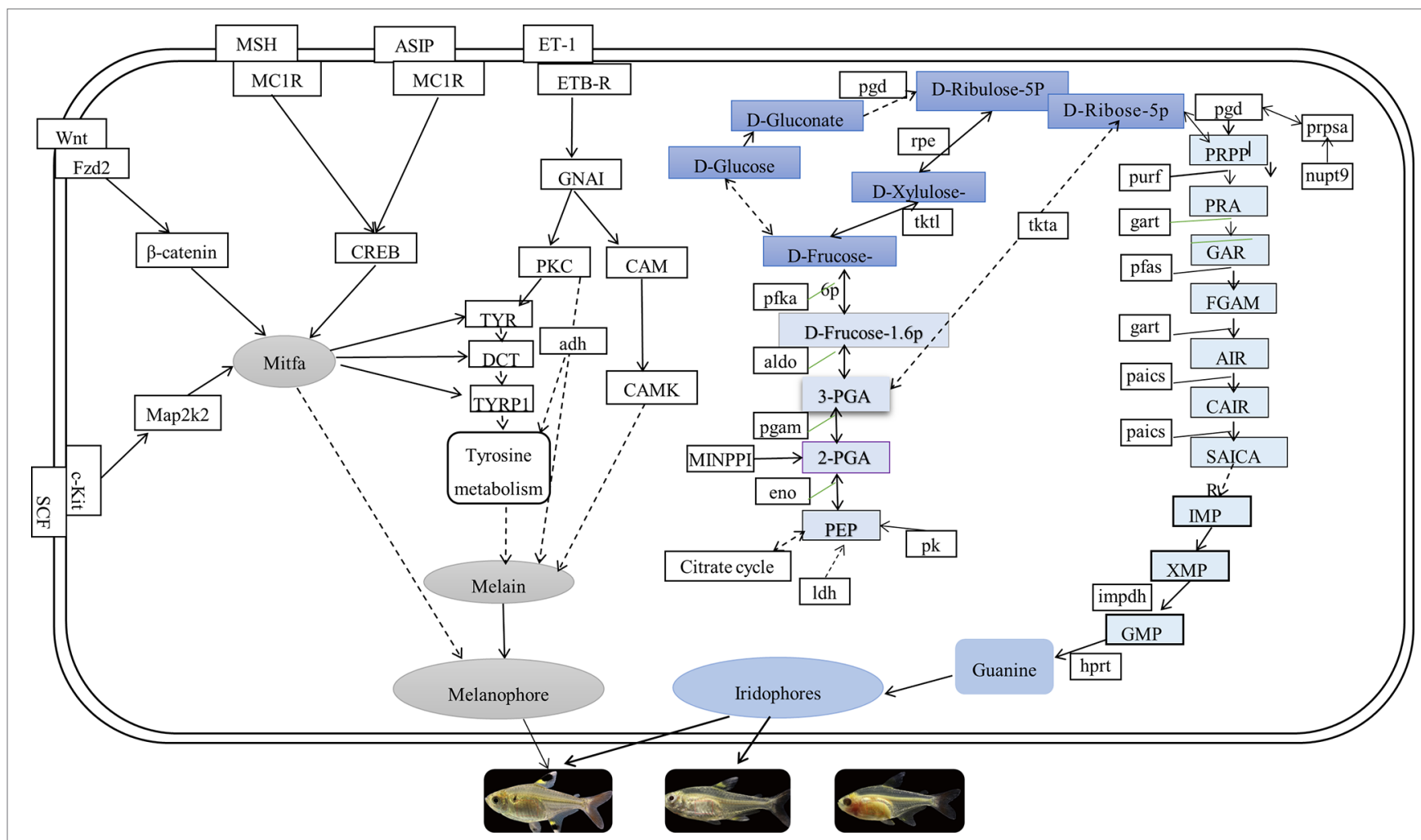

FIGURE 8 | The putative genes and pathways involved in pigmentation process of three phenotypic of Pristella maxillaris based on the present study.

synthase increases the number of iridophores ( $\mathrm{Ng}$ et al., 2009). In addition, Higdon et al. (2013) illustrated that specific enzymes such as aldo, eno, pgam1, and gart could regulate guanine synthesis. These results revealed the conservation of pigmentation genes across various species in terms of their sequences and functions. However, further investigations are still needed to determine how these genes work together to regulate guanine synthesis in iridophores.

To test the reliability of the RNA-Seq data, nine genes were randomly selected for qRT-PCR, including chaperonin containing tcp1 subunit 3 (cct3), solute carrier family 25 member 33 (slc25a33), glutathione s-transferase ( $g s t$ ), and map2k2, and so on. The expression pattern of these pigment-specific genes by qRT-PCR coincided with the results of the RNA-Seq analysis, except for the $p k$, cam, and $t k t l$ genes. The expression levels measured using the two methods were roughly coincided, indicating the reliability of our transcriptome data. We found that body coloration differs among varieties and the distribution of chromatophores at the cellular level. Therefore, an increase in body transparency might be caused by the absence of melanophores and iridophores in P. maxillaris. Moreover, after analyzing the WT and two mutant transcriptomes, we found that differentially expressed candidate pigmentation genes mainly enriched pathways related to melanin and guanine synthesis. However, further work is still needed to determine how these pathways and genes regulate the development of melanophores and iridophores in the body transparency phenotypes of $P$. maxillaris.
In addition, we also found that most DEGs were enriched in ribosome-related pathways in the skin and peritoneal tissues of fish exhibiting different phenotypes of $P$. maxillaris, which indicated that ribosomes might play an important role in fish body color formation. Higdon et al. (2013) found that four of the five most highly expressed genes were encoding ribosomal proteins in the transcriptome of zebrafish pigment cells. A similar finding was also reported in the transcriptome analysis of sheep skin (Ovis aries) (Fan et al., 2013). Some studies have proven that highly expressed levels of ribosome protein-related genes are correlated to black coat color in mice (Skarnes et al., 2011). Combined with the transcriptome data, we noted that the ribosomal protein genes might be involved in the formation of body coloration in $P$. maxillaris. However, further studies are needed to elucidate its exact function. We also discovered that some DEGs are involved in nucleotide excision repair, mismatch repair, oxidative phosphorylation, and systemic lupus erythematosus signaling pathways. These genes were significantly downregulated in the MU1 and MU2 fish compared with their expression in WT fish, which might be related to the absence of melanocytes and iridophores. Some studies have indicated that melanin from melanocytes not only scatters and absorbs UV as a physical barrier but also protects other epidermal cells by transferring melanin and reducing DNA damage (Kobayashi et al., 1998; Brenner and Hearing, 2008). In addition, the scattered reflectors and the arbitrary orientations of iridophores reflected all wavelengths of light (Grether et al., 2004). 
Taken together, we observed significant differences in the types and distribution of pigment cells in three different phenotypes of $P$. maxillaris and elucidated the potential genes and signaling pathways involved in body transparency.

\section{DATA AVAILABILITY}

This manuscript contains previously unpublished data. The name of the accession number is PRJNA525550 (https://www.ncbi. nlm.nih.gov/bioproject/PRJNA525550).

\section{AUTHOR CONTRIBUTIONS}

FB, XY, RY, and TC contributed to the study design, the major acquisition, analysis, and interpretation of data, and drafting/ revising the article. $\mathrm{FB}, \mathrm{ZO}$, and $\mathrm{JL}$ performed most of the laboratory work, and BT and MY assisted. FB contributed to the analysis of the data and wrote the manuscript. All authors read and approved the final manuscript.

\section{REFERENCES}

Bagnara, J. T., Fernandez, P. J., and Fujii, R. (2007). On the blue coloration of vertebrates. Pigment Cell Res. 20, 14-26. doi: 10.1111/j.1600-0749.2006.00360.x

Braasch, I., Schartl, M., and Volff, J. N. (2007). Evolution of pigment synthesis pathways by gene and genome duplication in fish. BMC Evol. Biol. 7, 74. doi: 10.1186/1471-2148-7-74

Brenner, M., and Hearing, V. J. (2008). The protective role of melanin against UV damage in human skin. Photochem. Photobiol. 84, 539-549. doi: 10.1111/ j.1751-1097.2007.00226.x

Busca, R., and Ballotti, R. (2000). Cyclic AMP a key messenger in the regulation of skin pigmentation. Pigment Cell Res. 13, 60-69. doi: 10.1034/j.1600-0749.2000.130203.x

Cheli, Y., Ohanna, M., Ballotti, R., and Bertolotto, C. (2010). Fifteen-year quest for microphthalmia-associated transcription factor target genes. Pigment Cell Melanoma Res. 23, 27-40. doi: 10.1111/j.1755-148X.2009.00653.x

Croucher, P. J., Brewer, M. S., Winchell, C. J., Oxford, G. S., and Gillespie, R. G. (2013). De novo characterization of the gene-rich transcriptomes of two color-polymorphic spiders, Theridion grallator and T. californicum (Araneae: Theridiidae), with special reference to pigment genes. BMC Genomics 14, 862 . doi: 10.1186/1471-2164-14-862

Darias, M. J., Andree, K. B., Boglino, A., Rotllant, J., Cerda-Reverter, J. M., Estevez, A., et al. (2013). Morphological and molecular characterization of dietary-induced pseudo-albinism during post-embryonic development of Solea senegalensis (Kaup, 1858). PLoS One 8, e68844. doi: 10.1371/journal.pone.0068844

Dooley, C. M., Mongera, A., Walderich, B., and Nusslein-Volhard, C. (2013). On the embryonic origin of adult melanophores: the role of ErbB and Kit signalling in establishing melanophore stem cells in zebrafish. Development 140, 10031013. doi: 10.1242/dev.087007

Failde, L. D., Bermudez, R., Vigliano, F., Coscelli, G. A., and Quiroga, M. I. (2014). Morphological, immunohistochemical and ultrastructural characterization of the skin of turbot (Psetta maxima L). Tissue Cell 46, 334-342. doi: 10.1016/j. tice.2014.06.004

Fan, R., Xie, J., Bai, J., Wang, H., Tian, X., Bai, R., et al. (2013). Skin transcriptome profiles associated with coat color in sheep. BMC Genomics 14, 389. doi: 10.1186/1471-2164-14-389

Fang, J., Chen, T., Pan, Q., and Wang, Q. (2018). Generation of albino medaka (Oryzias latipes) by CRISPR/Cas9. J. Exp. Zool. B Mol. Dev. Evol. 330, 242-246. doi: $10.1002 /$ jez.b.22808

Franco-Belussi, L., Nilsson Skold, H., and De Oliveira, C. (2016). Internal pigment cells respond to external UV radiation in frogs. J. Exp. Biol. 219, 1378-1383. doi: $10.1242 /$ jeb. 134973

\section{FUNDING}

The Huazhong Agricultural University Scientific \& Technological Self-Innovation Foundation (2662018PY083), the National Natural Science Foundation of China (31771648), and the Finance Special Fund of Ministry of Agricultural of China (Fisheries resources and environment survey in the key water areas of Tibet) supported this study.

\section{ACKNOWLEDGMENTS}

We thank LetPub (www.letpub.com) for its linguistic assistance during the preparation of this manuscript.

\section{SUPPLEMENTARY MATERIAL}

The Supplementary Material for this article can be found online at: https://www.frontiersin.org/articles/10.3389/fgene.2019.00698/ full\#supplementary-material

Fujimura, N., Taketo, M. M., Mori, M., Korinek, V., and Kozmik, Z. (2009). Spatial and temporal regulation of $\mathrm{Wnt} /$ beta-catenin signaling is essential for development of the retinal pigment epithelium. Dev. Biol. 334, 31-45. doi: 10.1016/j.ydbio.2009.07.002

Goda, M., Fujiyoshi, Y., Sugimoto, M., and Fujii, R. (2013). Novel dichromatic chromatophores in the integument of the mandarin fish Synchiropus splendidus. Biol. Bull. 224, 14-17. doi: 10.1086/BBLv224n1p14

Grabherr, M. G., Haas, B. J., Yassour, M., Levin, J. Z., Thompson, D. A., Amit, I., et al. (2011). Full-length transcriptome assembly from RNA-Seq data without a reference genome. Nat. Biotechnol. 29, 644-652. doi: 10.1038/nbt.1883

Grether, G. F., Kolluru, G. R., and Nersissian, K. (2004). Individual colour patches as multicomponent signals. Biol. Rev. Camb. Philos. Soc. 79, 583-610. doi: $10.1017 / \mathrm{S} 1464793103006390$

Henning, F., Jones, J. C., Franchini, P., and Meyer, A. (2013). Transcriptomics of morphological color change in polychromatic Midas cichlids. BMC Genomics 14, 171. doi: 10.1186/1471-2164-14-171

Henning, F., Renz, A. J., Fukamachi, S., and Meyer, A. (2010). Genetic, comparative genomic, and expression analyses of the Mclr locus in the polychromatic Midas cichlid fish (Teleostei, Cichlidae Amphilophus sp). species group. J. Mol. Evol. 70, 405-412. doi: 10.1007/s00239-010-9340-4

Higdon, C. W., Mitra, R. D., and Johnson, S. L. (2013). Gene expression analysis of zebrafish melanocytes, iridophores, and retinal pigmented epithelium reveals indicators of biological function and developmental origin. PLoS One 8, e67801. doi: 10.1371/journal.pone.0067801

Hirata, M., Nakamura, K., Kanemaru, T., Shibata, Y., and Kondo, S. (2003). Pigment cell organization in the hypodermis of zebrafish. Dev. Dyn. 227, 497503. doi: $10.1002 /$ dvdy. 10334

Hirata, M., Nakamura, K., and Kondo, S. (2005). Pigment cell distributions in different tissues of the zebrafish, with special reference to the striped pigment pattern. Dev. Dyn. 234, 293-300. doi: 10.1002/dvdy.20513

Hoekstra, H. E., Hirschmann, R. J., Bundey, R. A., Insel, P. A., and Crossland, J. P. (2006). A single amino acid mutation contributes to adaptive beach mouse color pattern. Science 313, 101-104. doi: 10.1126/science.1126121

Irion, U., Singh, A. P., and Nusslein-Volhard, C. (2016). The developmental genetics of vertebrate color pattern formation: lessons from zebrafish. Curr. Top. Dev. Biol. 117, 141-169. doi: 10.1016/bs.ctdb.2015.12.012

Jiang, Y., Zhang, S., Xu, J., Feng, J., Mahboob, S., Al-Ghanim, K. A., et al. (2014). Comparative transcriptome analysis reveals the genetic basis of skin color variation in common carp. PLoS One 9, e108200. doi: 10.1371/journal.pone.0108200

Kelsh, R. N. (2004). Genetics and evolution of pigment patterns in fish. Pigment Cell Res. 17, 326-336. doi: 10.1111/j.1600-0749.2004.00174.x 
Kimura, T., Takehana, Y., and Naruse, K. (2017). pnp4a Is the causal gene of the Medaka Iridophore mutant guanineless. G3 (Bethesda) 7, 1357-1363. doi: $10.1534 / \mathrm{g} 3.117 .040675$

Kobayashi, N., Nakagawa, A., Muramatsu, T., Yamashina, Y., Shirai, T., Hashimoto, M. W., et al. (1998). Supranuclear melanin caps reduce ultraviolet induced DNA photoproducts in human epidermis. J. Invest. Dermatol. 110, 806-810. doi: 10.1046/j.1523-1747.1998.00178.x

Krauss, J., Astrinidis, P., Frohnhofer, H. G., Walderich, B., and Nusslein-Volhard, C. (2013). Erratum: transparent, a gene affecting stripe formation in Zebrafish, encodes the mitochondrial protein Mpv17 that is required for iridophore survival. Biol. Open 2, 979. doi: 10.1242/bio.20136239

Leclercq, E., Taylor, J. F., and Migaud, H. (2010). Morphological skin colour changes in teleosts. Fish Fish. (Oxf) 11, 159-193. doi: 10.1111/j.1467-2979.2009.00346.x

Levy, C., Khaled, M., and Fisher, D. E. (2006). MITF: master regulator of melanocyte development and melanoma oncogene. Trends Mol. Med. 12, 406414. doi: 10.1016/j.molmed.2006.07.008

Li, S., Wang, C., Yu, W., Zhao, S., and Gong, Y. (2012). Identification of genes related to white and black plumage formation by RNA-Seq from white and black feather bulbs in ducks. PLoS One 7, e36592. doi: 10.1371/journal.pone.0036592

Li, X. M., Song, Y. N., Xiao, G. B., Zhu, B. H., Xu, G. C., Sun, M. Y., et al. (2015). Gene expression variations of red-white skin coloration in common carp (Cyprinus carpio). Int. J. Mol. Sci. 16, 21310-21329. doi: 10.3390/ijms160921310

Lowe, C., and Goodman-Lowe, G. (1996). Suntanning in hammerhead sharks. Nature 383, 677. doi: 10.1038/383677a0

Ma, D., Yang, H., Sun, L., and Xu, D. (2014). Comparative analysis of transcriptomes from albino and control sea cucumbers, Apostichopus japonicus. Acta Oceanol. Sin. 33, 55-61. doi: 10.1007/s13131-014-0464-z

Mao, X., Cai, T., Olyarchuk, J. G., and Wei, L. (2005). Automated genome annotation and pathway identification using the KEGG Orthology (KO) as a controlled vocabulary. Bioinformatics 21, 3787-3793. doi: 10.1093/bioinformatics/bti430

Michiels, N. K., Anthes, N., Hart, N. S., Herler, J., Meixner, A. J., Schleifenbaum, F., et al. (2008). Red fluorescence in reef fish: a novel signalling mechanism? BMC Ecol. 8, 16. doi: 10.1186/1472-6785-8-16

Muske, L. E., and Fernald, R. D. (1987). Control of a teleost social signal. II. Anatomical and physiological specializations of chromatophores. J. Comp. Physiol. A 160, 99-107. doi: 10.1007/BF00613445

Newton, R. A., Smit, S. E., Barnes, C. C., Pedley, J., Parsons, P. G., and Sturm, R. A. (2005). Activation of the cAMP pathway by variant human MC1R alleles expressed in HEK and in melanoma cells. Peptides 26, 1818-1824. doi: 10.1016/j.peptides.2004.11.031

Ng, A., Uribe, R. A., Yieh, L., Nuckels, R., and Gross, J. M. (2009). Zebrafish mutations in gart and paics identify crucial roles for de novo purine synthesis in vertebrate pigmentation and ocular development. Development 136, 26012611. doi: $10.1242 / \mathrm{dev} .038315$

Nilsson Skold, H., Aspengren, S., and Wallin, M. (2013). Rapid color change in fish and amphibians - function, regulation, and emerging applications. Pigment Cell Melanoma Res. 26, 29-38. doi: 10.1111/pcmr.12040

Nilsson Skold, H., Svensson, P. A., and Zejlon, C. (2010). The capacity for internal colour change is related to body transparency in fishes. Pigment Cell Melanoma Res. 23, 292-295. doi: 10.1111/j.1755-148X.2010.00674.X

Opdecamp, K., Nakayama, A., Nguyen, M. T., Hodgkinson, C. A., Pavan, W. J., and Arnheiter, H. (1997). Melanocyte development in vivo and in neural crest cell cultures: crucial dependence on the Mitf basic-helix-loop-helix-zipper transcription factor. Development 124, 2377-2386. https://dev.biologists.org/ content/develop/124/12/2377.full.pdf

Parichy, D. M. (2006). Evolution of danio pigment pattern development. Heredity (Edinb) 97, 200-210. doi: 10.1038/sj.hdy.6800867

Parichy, D. M. (2007). Homology and the evolution of novelty during Danio adult pigment pattern development. J. Exp. Zool. B Mol. Dev. Evol. 308, 578-590. doi: 10.1002/jez.b.21141

Park, H. Y., Wu, C., Yonemoto, L., Murphy-Smith, M., Wu, H., Stachur, C. M., et al. (2006). MITF mediates cAMP-induced protein kinase C-beta expression in human melanocytes. Biochem. J. 395, 571-578. doi: 10.1042/BJ20051388

Roberts, R. B., Ser, J. R., and Kocher, T. D. (2009). Sexual conflict resolved by invasion of a novel sex determiner in Lake Malawi cichlid fishes. Science 326, 998-1001. doi: 10.1126/science.1174705

Robinson, M. D., Mccarthy, D. J., and Smyth, G. K. (2010). edgeR: a Bioconductor package for differential expression analysis of digital gene expression data. Bioinformatics 26, 139-140. doi: 10.1093/bioinformatics/btp616
Sakai, C., Ollmann, M., Kobayashi, T., Abdel-Malek, Z., Muller, J., Vieira, W. D., et al. (1997). Modulation of murine melanocyte function in vitro by agouti signal protein. EMBO J. 16, 3544-3552. doi: 10.1093/emboj/16.12.3544

Schartl, M., Larue, L., Goda, M., Bosenberg, M. W., Hashimoto, H., and Kelsh, R. N. (2016). What is a vertebrate pigment cell? Pigment Cell Melanoma Res. 29, 8-14. doi: $10.1111 / \mathrm{pcmr} .12409$

Sefc, K. M., Brown, A. C., and Clotfelter, E. D. (2014). Carotenoid-based coloration in cichlid fishes. Comp. Biochem. Physiol. A Mol. Integr. Physiol. 173C, 42-51. doi: 10.1016/j.cbpa.2014.03.006

Shannon, P., Markiel, A., Ozier, O., Baliga, N. S., Wang, J. T., Ramage, D., et al. (2003). Cytoscape: a software environment for integrated models of biomolecular interaction networks. Genome Res. 13, 2498-2504. doi: 10.1101/gr.1239303

Skarnes, W. C., Rosen, B., West, A. P., Koutsourakis, M., Bushell, W., Iyer, V., et al. (2011). A conditional knockout resource for the genome-wide study of mouse gene function. Nature 474, 337-342. doi: 10.1038/nature10163

Voisey, J., Box, N. F., and Van Daal, A. (2001). A polymorphism study of the human Agouti gene and its association with MC1R. Pigment Cell Res. 14, 264-267. doi: 10.1034/j.1600-0749.2001.140406.x

Wang, C., Wachholtz, M., Wang, J., Liao, X., and Lu, G. (2014). Analysis of the skin transcriptome in two oujiang color varieties of common carp. PLoS One 9 , e90074. doi: 10.1371/journal.pone.0090074

White, R. M., Sessa, A., Burke, C., Bowman, T., Leblanc, J., Ceol, C., et al. (2008). Transparent adult zebrafish as a tool for in vivo transplantation analysis. Cell Stem Cell 2, 183-189. doi: 10.1016/j.stem.2007.11.002

Wucherer, M. F., and Michiels, N. K. (2012). A fluorescent chromatophore changes the level of fluorescence in a reef fish. PLoS One 7, e37913. doi: 10.1371/journal. pone. 0037913

Wucherer, M. F., and Michiels, N. K. (2014). Regulation of red fluorescent light emission in a cryptic marine fish. Front. Zool. 11, 1. doi: 10.1186/1742-9994-11-1

Xing, Y., Xu, W., Yang, K., Lian, X., and Yang, T. (2011). Immunolocalization of Wnt5a during the hair cycle and its role in hair shaft growth in mice. Acta Histochem. 113, 608-612. doi: 10.1016/j.acthis.2010.06.006

Yamaguchi, T. P., Bradley, A., Mcmahon, A. P., and Jones, S. (1999). A Wnt5a pathway underlies outgrowth of multiple structures in the vertebrate embryo. Development 126, 1211-1223. https://dev.biologists.org/content/develop/126/6/1211.full.pdf

Ye, J., Fang, L., Zheng, H., Zhang, Y., Chen, J., Zhang, Z., et al. (2006). WEGO: a web tool for plotting GO annotations. Nucleic Acids Res. 34, W293-W297. doi: 10.1093/nar/gkl031

Young, M. D., Wakefield, M. J., Smyth, G. K., and Oshlack, A. (2010). Gene ontology analysis for RNA-seq: accounting for selection bias. Genome Biol. 11, R14. doi: 10.1186/gb-2010-11-2-r14

Yu, L.-W., Zhang, M.-R., Liu, C.-H., Wang, Y.-H., and Liu, Z.-W. (2018), Karyotyping the Pristella Tetra (Pristella maxillaris). Chin. J Zool 53, 99-105. http://en.cnki.com.cn/Article_en/CJFDTotal-BIRD201801017.htm

Zeng, Z., Johnson, S. L., Lister, J. A., and Patton, E. E. (2015). Temperature-sensitive splicing of mitfa by an intron mutation in zebrafish. Pigment Cell Melanoma Res. 28, 229-232. doi: 10.1111/pcmr.12336

Zhang, Y., Liu, J., Peng, L., Ren, L., Zhang, H., Zou, L., et al. (2017). Comparative transcriptome analysis of molecular mechanism underlying gray-to-red body color formation in red crucian carp (Carassius auratus, red var). Fish Physiol. Biochem. 43, 1387-1398. doi: 10.1007/s10695-017-0379-7

Zhang, Y. P., Wang, Z. D., Guo, Y. S., Liu, L., Yu, J., Zhang, S., et al. (2015). Morphological characters and transcriptome profiles associated with black skin and red skin in crimson snapper (Lutjanus erythropterus). Int. J. Mol. Sci. 16, 26991-27004. doi: 10.3390/ijms161126005

Zhu, W., Wang, L., Dong, Z., Chen, X., Song, F., Liu, N., et al. (2018). Erratum: comparative transcriptome analysis identifies candidate genes related to skin color differentiation in red tilapia. Sci Rep. 8, 46979. doi: 10.1038/srep46979

Conflict of Interest Statement: The authors declare that the research was conducted in the absence of any commercial or financial relationships that could be construed as a potential conflict of interest.

Copyright $\odot 2019$ Bian, Yang, Ou, Luo, Tan, Yuan, Chen and Yang. This is an openaccess article distributed under the terms of the Creative Commons Attribution License (CC BY). The use, distribution or reproduction in other forums is permitted, provided the original author(s) and the copyright owner(s) are credited and that the original publication in this journal is cited, in accordance with accepted academic practice. No use, distribution or reproduction is permitted which does not comply with these terms. 